\title{
Theoretical and experimental study of the transport of granular materials by inclined vibratory conveyors
}

\author{
E.M. Sloot, N.P. Kruyt \\ Department of Mechanical Engineering. University of Twente, PO Box 217, 7500 AE Enschede, Netherlands
}

Received 22 March 1995; revised 25 October 1995

\begin{abstract}
A theoretical and experimental study was made of the conveying speed with which granular materials are transported by vibratory conveyors. The basic assumption made is that the layer of granular material can be considered as a point mass. The theory incorporates rest, slide, and flight phases of the material. Although the emphasis of this study is on the effect of the inclination (and declination) of the conveyor on the conveying speed, the effects of throw number, friction coefficient and vibration angle on the conveying speed are also shown. A useful method is presented for measuring the coefficient of friction between granular material and vibratory conveyor. Experiments were performed in order to verify the point mass theory. The agreement between theory and experiment is fairly good for slide conveyors but for throw conveyors larger deviations are observed. Some possibilities for improvement to the theory are briefly investigated.
\end{abstract}

Keywords: Friction; Granular material; Vibratory conveyor

\section{Introduction}

Vibratory conveyors are often used in industry to transport granular materials. They consist of a trough which is (generally) vibrated sinusoidally in time. This vibration induces the movement of the granular material along the conveyor surface. The direction, amplitude and frequency of the oscillations are design parameters of the conveyor, see Fig. 1.

Some of the main advantages of vibratory conveyors are their simple construction, their suitability to handle hot and abrasive materials and their applicability as dosing equipment. Since the trough can be totally enclosed, they are also well suited to the transport of dusty materials. Some disadvantages of vibratory conveyors are their noisy operation, the induced vibrations on their surroundings and their limited transport distance. Furthermore, the granular material may be damaged when it is subjected to large accelerations normal to the trough.

A distinction can be made between slide and throw conveyors: for slide conveyors the material remains in contact with the trough surface, while for throw conveyors the material loses contact during part of the conveying cycle. Sometimes the conveyors are positioned under a small inclination or declination instead of horizontally. It is expected that this trough slope will have a large influence on the conveying speed, especially for slide conveyors. This influence constitutes an important subject of this study.
Many researchers investigated the transport of granular materials by vibratory conveyors. Booth and McCallion [1] made a theoretical and experimental study of the conveying speed of slide conveyors. Nedderman and Harding [2] extended this analysis and presented some optimization studies for horizontal and inclined sliding. Pajer et al. [3] dealt with practical aspects of slide and throw conveyors. Erdesz and Szalay [4], Erdesz and Németh [5] made a practical and theoretical study of slide and throw conveyors. Some theoretical optimization studies were performed by Hota and Karmaker [6]. The most extensive research was reported by Rademacher and ter Borg [7,8]. They presented a point mass theory for horizontal vibratory conveyors as well as the results of a large number of experiments that were performed on several types of horizontal conveyors transporting many different granular materials. Some purely numerical studies were done by $\mathrm{Ng}$ et al. [9] and Lim [10]. The experimental

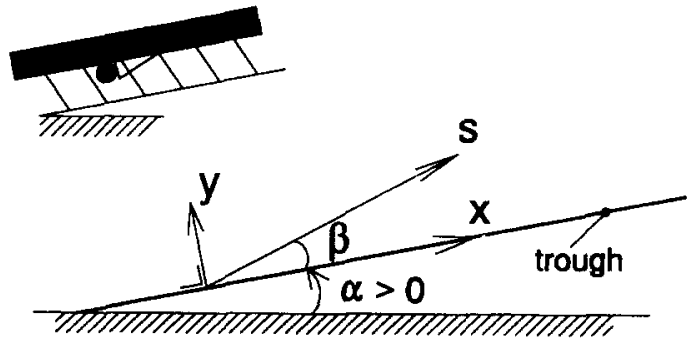

Fig. 1. Lay-out of the vibratory conveyor. 
verifications in Ref. [9] were restricted to a single vibration angle. A special type of vibratory conveyor, a corrugated vibratory conveyor, is described by Persson and Megnin [11].

The emphasis of the present theoretical and experimental study is on inclined and declined transport, both for slide and throw conveyors. In contrast with $\mathrm{Ng}$ et al. [9] and Lim [10], the theory in this article is largely analytical, in order to gain more insight into the occurring phases of motion of the granular material.

The basic assumptions in this theory are:

(i) the trough is driven fully sinusoidally;

(ii) the granular material is assumed to behave like a point mass, it moves like a rigid body with negligible rotations;

(iii) a fully plastic collision is assumed when the layer of granular material hits the trough surface after a flight phase;

(iv) the distinction between the static and kinetic coefficient of friction is neglected, in contrast with Ref. [2];

(v) friction between the granular material and side walls of the trough is neglected,

(vi) air drag is neglected.

\section{Definitions}

The $x$ - and $y$-coordinates are defined tangential respectively normal to the trough (conveyor surface), see also Fig. 1 . The conveyor is oriented at an inclination angle $\alpha$ with respect to the horizontal; $\alpha<0$ denotes a declination. The coordinate $s$ is in the direction of vibration; the angle between the coordinate $s$ and the trough is the vibration angle $\beta$. The trough is assumed to be driven sinusoidally in time with angular velocity $\omega$ and amplitude $r$ :

$X_{\mathrm{t}}(t)=\frac{x_{\mathrm{t}}}{r \cos \beta}=\sin \omega t$

$\dot{X}_{\mathrm{t}}(t)=\cos \omega t \quad \ddot{X}_{\mathrm{t}}(t)=-\sin \omega t$

$Y_{\mathrm{t}}(t)=\frac{y_{\mathrm{t}}}{r \sin \beta}=\sin \omega t$

$\dot{Y}_{\mathrm{t}}(t)=\cos \omega t \quad \ddot{Y}_{\mathrm{t}}(t)=-\sin \omega t$

where $x_{\mathrm{t}}$ and $y_{\mathrm{t}}$ are the tangential respectively normal trough displacements. The dimensionless displacements, velocities and accelerations are denoted by symbols in capitals.

The throw number $\Gamma$ is defined as the dimensionless maximum acceleration normal to the trough:

$\Gamma \equiv \frac{\ddot{y}_{\mathrm{t}, \max }}{g \cos \alpha}=\frac{\omega^{2} r \sin \beta}{g \cos \alpha}$

For $\Gamma<1$ the material will remain in contact with the trough surface; for $\Gamma>1$ the material will lose contact with the trough and a flight phase occurs. Thus slide conveyors are charac- terized by $\Gamma<1$, while flight conveyors are characterized by $\Gamma>1$.

The machine number is defined as the dimensionless maximum trough acceleration:

$K \equiv \frac{\ddot{s}_{\max }}{g}=\frac{\omega^{2} r}{g}$

In practice the machine number will be restricted in order not to damage the granular material.

The velocity efficiency, which is a dimensionless measure of the conveying speed, is defined as the ratio of the average relative speed and the maximum tangential trough velocity:

$\eta \equiv \frac{\overline{\dot{x}}_{\mathrm{rel}}}{\omega r \cos \beta}=\frac{X_{\mathrm{rel}}}{2 \pi}$

In this expression $X_{\mathrm{rel}}=\left(X_{\mathrm{m}}-X_{\mathrm{t}}\right)$ denotes the relative material displacement during one cycle of $T=2 \pi / \omega$. The subscript $\mathrm{m}$ is used to denote the point mass.

\section{Theory}

During one cycle of the motion of the trough, the point mass may be in a rest, slide or flight phase, depending on the actual accelerations, velocities and displacements of the point mass and trough. In this section these various phases of motion are analysed.

The equations of motion of the point mass $m$ are:

$\mp F_{\mathrm{w}}-m g \sin \alpha=m \ddot{x}_{\mathrm{m}}$

$N-m g \cos \alpha=m \ddot{y}_{\mathrm{m}}$

Here, the upper or lower sign is used for sliding in the positive or negative $x$-direction. The maximum friction force is $F_{\mathrm{w}}=\mu N$, where $\mu$ is the coefficient of friction between granular material and trough.

\subsection{Slide and rest phases}

A slide phase will occur when the tangential acceleration of the trough is larger than that of the point mass. The slide phase is called positive when the displacement of the point mass is in the positive tangential direction relative to the trough and negative otherwise. A rest phase occurs when the relative tangential displacement is zero.

From Eq. (5) and $\ddot{y}_{\mathrm{m}}=\ddot{y}_{\mathrm{t}}$, the dimensionless acceleration of the mass is obtained during a slide phase:

$\ddot{X}_{\mathrm{m}}(t)=\tan \beta\left[\frac{1}{\Gamma}(\mp \mu-\tan \alpha) \pm \mu \sin \omega t\right]$

Sliding may start when the accelerations of the mass and the trough are equal. This occurs when:

$\sin \omega t= \pm \frac{1}{\Gamma} \frac{(\mu \pm \tan \alpha) \tan \beta}{1 \pm \mu \tan \beta}$ 
where Eq. (1) was used. This implies that a positive or negative slide phase may occur within the interval $\left[\delta_{1}, \delta_{2}\right]$, respectively $\left[\delta_{3}, \delta_{4}\right]$ :

$\sin \delta_{1,2}=\left[\frac{1}{\Gamma} \frac{(\mu+\tan \alpha) \tan \beta}{1+\mu \tan \beta}\right]$

$\sin \delta_{3,4}=\left[-\frac{1}{\Gamma} \frac{(\mu-\tan \alpha) \tan \beta}{1-\mu \tan \beta}\right]$

A slide phase will actually begin at $\delta_{i}(i=1,3)$ only if the previous phase has ended.

The velocity of the point mass is obtained by integrating Eq. (6) in time. The employed boundary condition is that the velocities of point mass and trough are equal at the start of a positive or negative slide phase. This start occurs at $\omega t=$ $\delta_{\mathrm{P} / \mathrm{N}}$. The dimensionless velocity of the point mass is then given by:

$$
\begin{aligned}
& \dot{X}_{\mathrm{m}, \mathrm{P} / \mathrm{N}}(t)=\mp \mu\left(\cos \omega t-\cos \delta_{\mathrm{P} / \mathrm{N}}\right) \tan \beta \\
& \quad \mp \frac{1}{\Gamma} \tan \beta(\mu \pm \tan \alpha)\left(\omega t-\delta_{\mathrm{P} / \mathrm{N}}\right)+\cos \delta_{\mathrm{P} / \mathrm{N}}
\end{aligned}
$$

Integrating Eq. (9) from $\delta_{\mathrm{P} / \mathrm{N}}$ to $\epsilon_{\mathrm{P} / \mathrm{N}}$ and using Eqs. (1) and (8), the dimensionless relative displacement becomes:

$$
\begin{aligned}
X_{\mathrm{rel}, \mathrm{P} / \mathrm{N}}= & (1 \pm \mu \tan \beta)[\sin \delta-\sin \epsilon \\
& \left.+\cos \delta(\epsilon-\delta)-1 / 2(\epsilon-\delta)^{2} \sin \delta_{i}\right]
\end{aligned}
$$

In this equation the subscripts $\mathrm{P} / \mathrm{N}$ have been omitted for $\delta$ and $\epsilon$. This expression is used to calculate the velocity efficiency for positive and negative slide phases.

The end of a slide phase, $\epsilon$, is defined as the time at which mass and trough are moving with the same tangential velocity. Using Eqs. (1), (8) and (9) it follows:

$\frac{\cos \epsilon-\cos \delta}{\epsilon-\delta}=-\sin \delta_{i}$

In this expression $i=1$ indicates a positive slide phase and $i=3$ a negative slide phase. The derivation of this equation is also shown in Refs. [1,2 and 8].

A rest phase will occur if a slide phase has ended before the next phase starts.

\subsection{Flight phase}

A flight phase occurs if the normal force $N$ becomes zero. At the start of a flight the mass and trough have the same $y$ coordinate and $y$-velocity. From Eqs. (1) and (5) it follows that the flight phase starts at $\omega t=\delta_{\mathrm{F}}$, where:

$\delta_{\mathrm{F}}=\arcsin (1 / \Gamma)$

During the flight the normal component of the acceleration acting on the point mass remains $-g \cos \alpha$. The normal displacement can be determined by integrating with the boundary conditions of equal normal coordinates and velocities at $\omega t=\delta_{\mathrm{F}}$ :
$Y_{\mathrm{m}}(t)=-\frac{1}{2 \Gamma}\left(\omega t-\delta_{\mathrm{F}}\right)^{2}+\sin \delta_{\mathrm{F}}+\cos \delta_{\mathrm{F}}\left(\omega t-\delta_{\mathrm{F}}\right)$

The end of the flight is derived from the condition $Y_{\mathrm{m}}\left(\epsilon_{\mathrm{F}}\right)=Y_{\mathrm{t}}\left(\epsilon_{\mathrm{F}}\right)$, which yields an implicit equation in $\epsilon_{\mathrm{F}}$, using Eqs. (12) and (13):

$$
\begin{array}{r}
\sin \delta_{\mathrm{F}}-\sin \epsilon_{\mathrm{F}}+\cos \delta_{\mathrm{F}}\left(\epsilon_{\mathrm{F}}-\delta_{\mathrm{F}}\right) \\
-1 / 2 \sin \delta_{\mathrm{F}}\left(\epsilon_{\mathrm{F}}-\delta_{\mathrm{F}}\right)^{2}=0
\end{array}
$$

The end of the flight can be expressed in the number of periods per flight, $n: \epsilon_{\mathrm{F}}=\delta_{\mathrm{F}}+2 \pi n$. Combining this expression with Eqs. (12) and (14) yields:

$\Gamma=\left[\left(\frac{\cos 2 \pi n+2 \pi^{2} n^{2}-1}{2 \pi n-\sin 2 \pi n}\right)^{2}+1\right]^{1 / 2}$

This equation was also derived in Refs. [3,5 and 8]. In order to restrict the machine number, $n$ is often kept below 1 in practice, or $\Gamma \leq 3.297$.

Since air drag is neglected, the tangential acceleration acting on the point mass remains equal to $\ddot{x}_{\mathrm{m}}=-g \sin \alpha$, or $\ddot{X}_{\mathrm{m}}=-(1 / \Gamma) \tan \alpha \tan \beta$. The velocity of the point mass can be determined by integrating this expression with the boundary condition of a known tangential velocity $V_{\mathrm{F}}$ at $\epsilon_{\mathrm{F}}$. This velocity is determined from the occurring phase before the flight phase. The relative displacement is obtained by integrating from $\delta_{\mathrm{F}}$ to $\epsilon_{\mathrm{F}}$ and by subtracting the trough displacement from that of the point mass, according to Eq. (1). This results in:

$$
\begin{aligned}
X_{\text {rel. F }}= & -\frac{1}{2 \Gamma} \tan \beta \tan \alpha\left(\epsilon_{\mathrm{F}}-\delta_{\mathrm{F}}\right)^{2} \\
& +V_{\mathrm{F}}\left(\epsilon_{\mathrm{F}}-\delta_{\mathrm{F}}\right)-\left(\sin \epsilon_{\mathrm{F}}-\sin \delta_{\mathrm{F}}\right)
\end{aligned}
$$

This equation is used to calculate the velocity efficiency of a flight phase according to Eq. (4).

The change of the velocity of the point mass during a collision with the trough is related to the total linear impulse at time $\epsilon_{\mathrm{F}}$ by the linear momentum equation:

$$
\begin{aligned}
& \int_{\Delta t} N \mathrm{~d} t=m\left[\dot{y}_{\mathrm{m}}\left(\epsilon_{\mathrm{F}}^{+}\right)-\dot{y}_{\mathrm{m}}\left(\epsilon_{\mathrm{F}}^{-}\right)\right] \\
& \quad \mp \mu \int_{\Delta t} N \mathrm{~d} t=m\left[\dot{x}_{\mathrm{m}}\left(\epsilon_{\mathrm{F}}^{+}\right)-\dot{x}_{\mathrm{m}}\left(\epsilon_{\mathrm{F}}^{-}\right)\right]=m \Delta \dot{x}_{\mathrm{m}, \mathrm{C}}
\end{aligned}
$$

Due to the assumption of a fully plastic collision, the first term on the right-hand side of Eq. (17a) equals the trough velocity at $\epsilon_{\mathrm{F}}$. The sign in (17b) depends on the sign of the relative velocity. Combining these two equations gives the change of velocity during a collision:

$\Delta \dot{X}_{\mathrm{m}, \mathrm{C}}=\mp \mu \tan \beta\left[\cos \epsilon_{\mathrm{F}}-\cos \delta_{\mathrm{F}}+\frac{1}{\Gamma}\left(\epsilon_{\mathrm{F}}-\delta_{\mathrm{F}}\right)\right]$

Here the value of the normal velocity just before the collision $\left(\dot{y}_{\mathrm{m}}\left(\epsilon_{\mathrm{F}}^{-}\right)\right)$was obtained from Eq. (1) and the derivative of Eq. (13). It can be shown that the total change of velocity of the point mass during a flight and a collision is equal to the 
change of velocity during a positive or negative slide phase [8]. If the flight ends with a tangential velocity of the point mass that is not equal to that of the trough, a positive or negative slide phase occurs, depending on the sign of this difference, until the velocities become equal.

\subsection{Mode and velocity efficiency diagrams}

In order to calculate the occurring modes of motion and the velocity efficiencies, a computer program was developed to solve the presented equations. The occurring phases are indicated with $\mathrm{P}$ (positive slide), $\mathrm{N}$ (negative slide), $F$ (flight) and $\mathrm{P}^{\prime}$ and $\mathrm{N}^{\prime}$ for a positive or negative slide phase directly after a collision.

The main difference between this program and that of $\mathrm{Ng}$ [9] is that the current program calculates numerically only the beginning and end of each phase by means of the equations developed here. The program calculates the time instants at which the (potentially occurring) phases begin and end by iterating until a periodic solution with period $T=2 \pi / \omega$ is obtained. A detailed description of the program is given in Ref. [12].

In the case of horizontal transport ( $\alpha=0$ ), the occurring modes and velocity efficiency can be expressed as functions of $\mu \tan \beta$ and $\Gamma$ (see Ref. [8] for results). In Fig. 2 the occurring modes are shown for a declination with $\tan \alpha=-0.10$ and $\mu=0.35$. In Fig. 3 the velocity efficiency is plotted for the same declination and friction coefficient. In Fig. 4 the velocity efficiency is plotted for an inclination of $\tan \alpha=+0.10$ and $\mu=0.35$, and Fig. 5 shows the effect of the inclination on the velocity efficiency for common values of $\mu$ and $\beta$.

\subsection{Throw number for maximum velocity efficiency}

In this section the throw number is derived for which the velocity efficiency reaches its maximum. For a full cyclic flight the flight number $n$ equals $1 \quad(\Gamma=3.297)$. Then the tangential velocity of the point mass will not change in time in the case of horizontal transport: it follows from Eq. (12) that the velocity efficiency, $V_{\mathrm{F}}=\cos \left(\delta_{\mathrm{F}}\right)=0.954$ ( see the

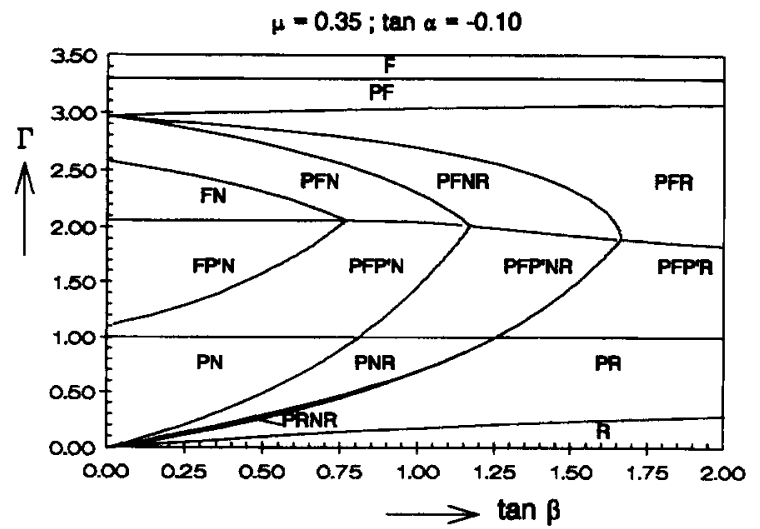

Fig. 2. Mode diagram of successive phases for declined transport ( $P=$ positive slide phase, $\mathrm{N}=$ negative slide phase, $\mathrm{R}=$ rest phase, $\mathrm{F}=$ flight phase, $P^{\prime}=$ positive slide phase after a collision, $N^{\prime}=$ negative slide phase after a collision).

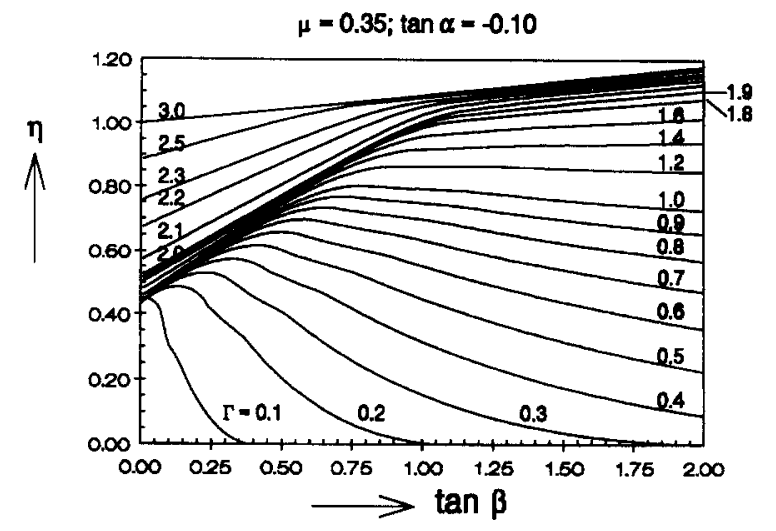

Fig. 3. Velocity efficiency for declined transport as a function of vibration angle $\beta$ and throw number $\Gamma$.

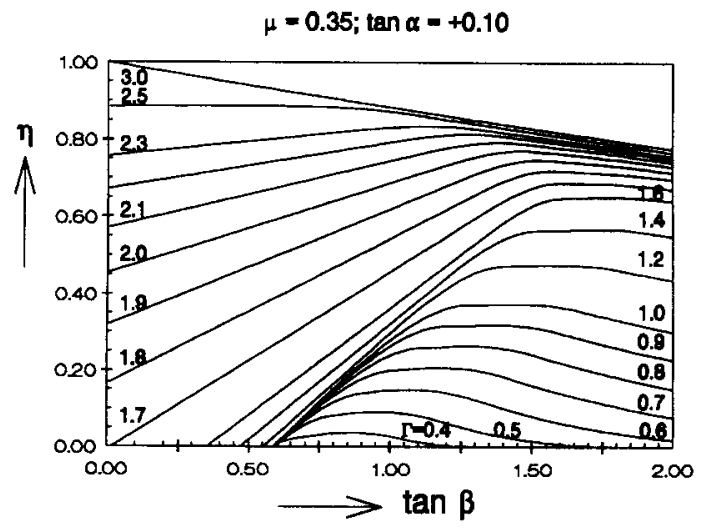

Fig. 4. Velocity efficiency for inclined transport as a function of vibration angle $\beta$ and throw number $\Gamma$.
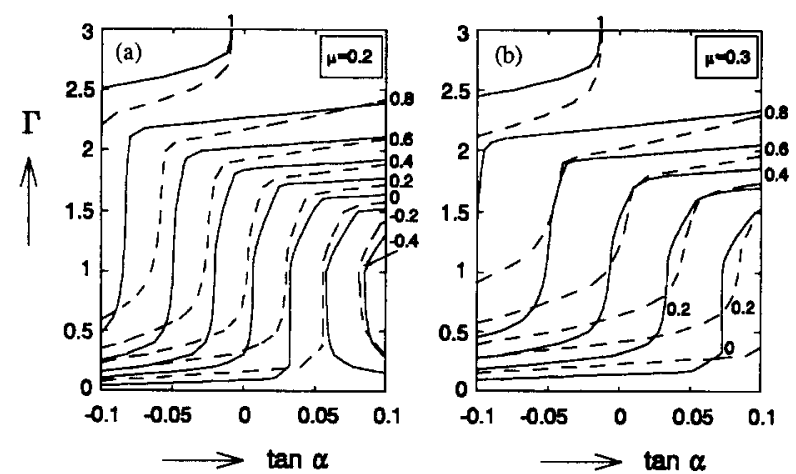

Fig. 5. Iso-curves of velocity efficiency as a function of inclination angle $\alpha$ and throw number $\Gamma$. (a) $\mu=0.2$; (b) $\mu=0.3 ;-, \beta=30^{\circ} ;---, \beta=45^{\circ}$.

horizontal line for $\Gamma=3.297$ in Fig. 6). In order to increase this efficiency, a small positive slide phase has to be present before the flight phase. The flight then starts at $\epsilon_{\mathrm{P}}=\delta_{\mathrm{F}}$ and ends at $\epsilon_{\mathrm{F}}=\delta_{\mathrm{p}}+2 \pi$. For a maximum dimensionless velocity of 1 at the start of the flight, $\beta$ must be equal to 0 according to Eqs. (8) and (9) with $\delta_{\mathrm{p}}=\delta_{1} \rightarrow 0$. The corresponding throw number is derived from Eq. (14), using $\epsilon_{\mathrm{F}}=\delta_{\mathrm{F}}+2 \pi n$ :

$\Gamma=\frac{2 \pi^{2} n^{2}-1}{2 \pi n \cos 2 \pi n}$

Together with Eq. (15) an implicit relation in $\Gamma$ is obtained; its solution is $\Gamma=2.975$ for each value of $\alpha$ and the maximum velocity efficiency is obtained for this throw number, see also 


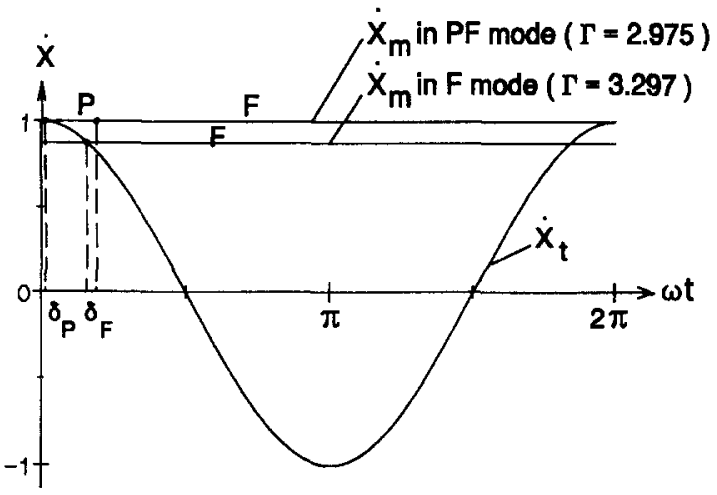

Fig. 6. Tangential velocities of trough and point mass as a function of time (large throw numbers).

Fig. 6. The dependence on $\mu$ and $\tan \beta$ seems to be very small, especially for horizontal transport [8]. Since the maximum velocity efficiency is obtained for $\Gamma=2.975$, it is not useful to design a vibratory conveyor with a throw number larger than 3.0.

\subsection{Limiting case of small vibration angles for slide phases}

In this section the case of a small vibration angle is studied in order to derive a relation that describes the influence of inclination on the velocity efficiency for small vibration angles. Furthermore, a method is described to measure the friction coefficient between granular material and trough.

Equations for the start and end of a $P N$ mode, which implies $\delta_{\mathrm{N}}=\epsilon_{\mathrm{P}}$ and $\epsilon_{\mathrm{N}}=\delta_{\mathrm{P}}+2 \pi$, are obtained from Eq. (11):

$\sin \left(\delta_{\mathrm{P}}+1 / 2 C\right)=\frac{(C-2 \pi) \sin \delta_{3}}{2 \sin (1 / 2 C)}$

$\epsilon_{\mathrm{P}}=C+\delta_{\mathrm{P}} \quad$ with $\quad C \equiv \frac{2 \pi}{1-\left(\sin \delta_{1}\right) /\left(\sin \delta_{3}\right)}$

This equation is valid for each value of $\beta$.

For $\beta \rightarrow 0$, two cases are now distinguished:

(i) $K<\infty$ : for this realistic case an elegant method was developed to obtain the primarily kinetic coefficient of friction of the granular material. This method is already mentioned in Ref. [8]; here it is described in detail. The trough has to move with a certain amplitude in purely horizontal direction $(\beta=0)$. In order to be certain that a PN mode develops for horizontal transport, $K$ must be larger than 0.67 for a friction coefficient that is smaller than 0.35 , as can be observed in the mode diagram in Fig. 7. By measuring either optically, or by using a felt pen, the maximum relative displacement between trough and an open box or cylinder filled with granular material, the coefficient of friction can be calculated implicitly from Eq. (10). The result is shown in Fig. 8.

(ii) $K \rightarrow \infty$ : this hypothetical case results in a very simple equation that demonstrates the influence of the inclination of the trough on the velocity efficiency for small vibration angles. In case of a $P N$ mode, which will always be the case

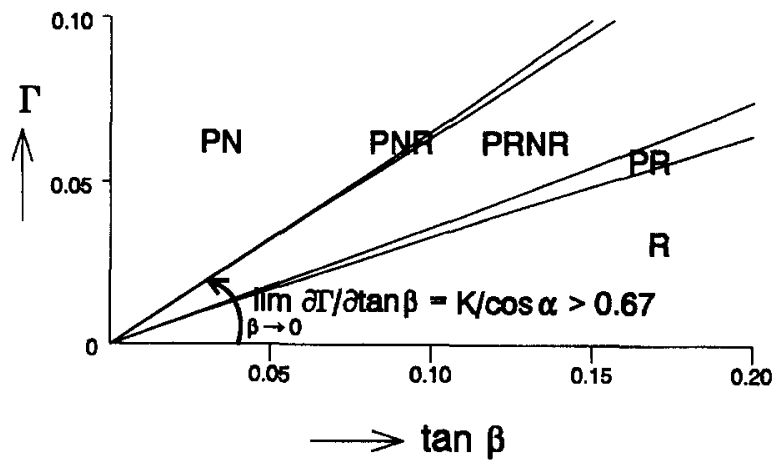

Fig. 7. Mode diagram for small vibration angles $(\alpha=0, \mu=0.35$ ).

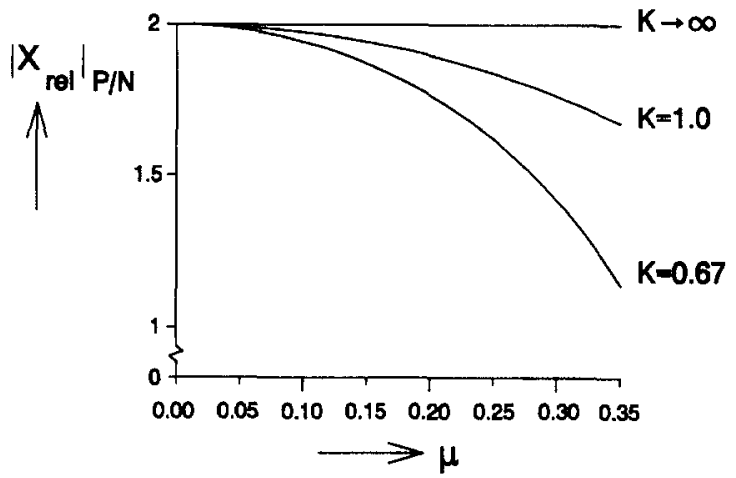

Fig. 8. Maximum relative displacement for a PN mode, as a function of friction coefficient $\mu$ and machine number $K$.

for infinite values of the machine number, it follows after some algebra from Eqs. (4), (8), (10) and (20) that the limiting value of the efficiency for each inclination or declination angle is given by:

$\eta_{\beta \rightarrow 0}=-\cos \left(\frac{C}{2}\right)=-\sin \left(\frac{\pi \tan \alpha}{2 \mu}\right)$

This expression is valid for $\Gamma \leq 1$. Note that the start of the positive slide phase is given by $\delta_{\mathrm{P}}=\pi-1 / 2 C$, according to Eq. (20) for $\beta \rightarrow 0$ and because $\delta_{\mathrm{p}} \epsilon\left[\delta_{1}, \delta_{2}\right]$. This equation also shows the antisymmetry between positive and negative slopes in case $\beta \rightarrow 0$. Eq. (21) predicts that the velocity efficiency equals 0.434 for $\beta \rightarrow 0$ and $\mu=0.35$; this is consistent with the results presented in Fig. 3.

\section{Experimental}

In order to verify the developed point mass theory, experiments were performed with an electrohydraulically driven vibratory conveyor. The exact dimensions of this $3 \mathrm{~m}$ long conveyor are given in Ref. [8]. The inclination of the conveyor was set by lifting it at one end over various heights. The trough was driven sinusoidally in time by means of one horizontal and two vertical hydraulic cylinders with electronic feed-back. By modifying the horizontal and vertical amplitudes and running frequency, the vibration angle and throw number were set. The velocity efficiency was determined by measuring the time that elapsed during the traverse of a marker in the granular material over a fixed distance. 


\subsection{Verifications of the assumptions}

With the experimental set-up, the following assumptions of the point mass theory were verified:

(a) by measuring the displacements in time of the trough with a digital data acquisition system it was verified that the displacements of the trough were sinusoidal.

(b) In order to investigate whether a vertical gradient of the velocity was present, a plastic straw was put into the granular material. Since the straw remained in its vertical position, it was concluded that no such gradient was present.

(c) The effect of the wall friction was investigated by distributing a number of straws over the width of the granular layer. Only a small influence of the wall friction was observed within a few millimetres distance from the walls, compared to the width of the trough of $200 \mathrm{~mm}$.

(d) For throw numbers $\Gamma \leq 2.5$ stable behaviour was observed. Measurements for larger throw numbers were more difficult to perform due to the presence of strong wiggles on the surface of the granular material.

\subsection{Measurements}

Measurements were performed with square poly(vinyl)chloride (PVC) grains $(\mu=0.23)$ and nearly round grains of spinach seed ( $\mu=0.24$ ), both for inclinations and declinations ( $\tan \alpha= \pm 0.02$ and $\tan \alpha= \pm 0.05$ ). The friction coefficients were measured using the method described in the previous section. Since no large differences were found between the velocity efficiencies of these two materials, only the results of the experiments with PVC grains are shown here for $\tan \alpha= \pm 0.05$. In Figs. 9 and 10 the results of the experiments and developed theory are compared. The total set of measurements can be obtained from the second author (NPK). As an extra check, measurements were also performed with a single wooden block with friction elements $(\mu=0.18)$ in sliding mode.

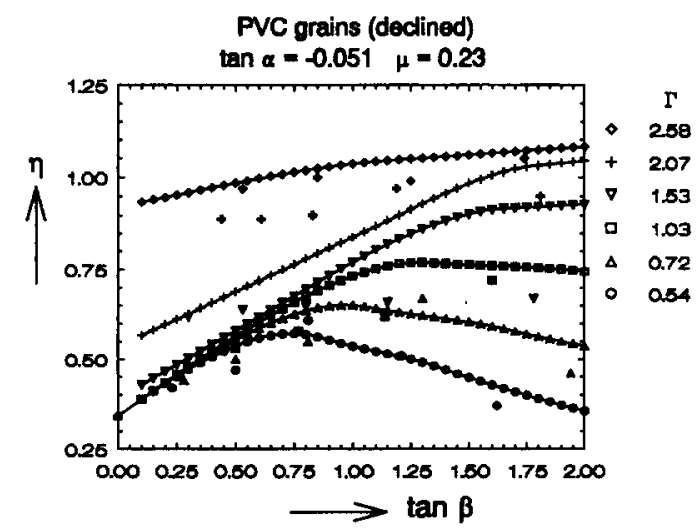

Fig. 9. Comparison of theoretical and experimental velocity efficiency for declined transport of PVC grains, $\tan \alpha=-0.051$; the open symbols stand for the theory and solid symbols for the experiments.

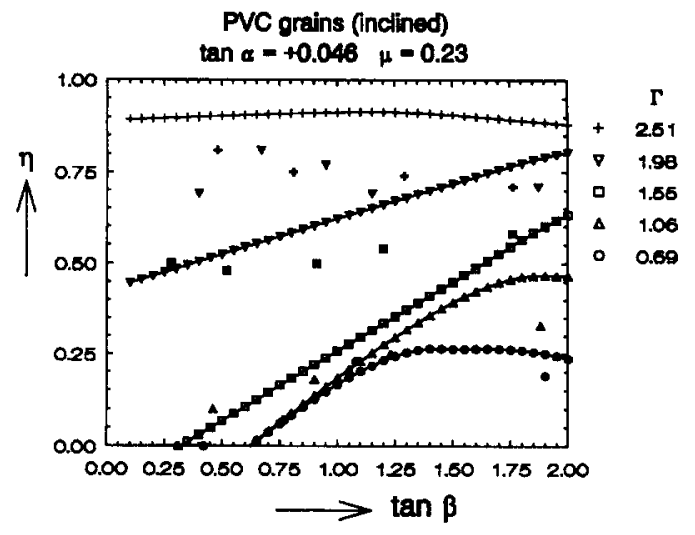

Fig. 10. Comparison of theoretical and experimental velocity efficiency for inclined transport of PVC grains, $\tan \alpha=+0.046$; the open symbols stand for the theory and solid symbols for the experiments.

\subsection{Comparison of theory and experiments}

Fairly good agreement between theory and experiment is found for slide conveyors $(\Gamma<1.0)$. Negative velocity efficiencies were not measured for granular materials in bulk, since the experimental set-up did not allow this. A single wooden block though did show negative efficiencies, as predicted by the theory. Overall, the velocity efficiencies of this single mass agrees within $20 \%$ with the theoretical predictions for $\Gamma<1$. For the granular materials, for large throw numbers ( $\Gamma \approx 2.5$ ) good agreement is obtained for negative slopes, but larger deviations are observed for positive slopes. The influence of vibration angle $\beta$ on the efficiency for throw numbers around 3.0 appears the same as at 2.5 , in agreement with the theory. Here reliable measurements were harder to perform due to the presence of wiggles on the surface of the granular material. For $\Gamma=1.5-2$ the theory predicts a large influence of the vibration angle on the velocity efficiency. However, the results of the experiments do not exhibit this large influence. A similar order of discrepancy was found by Rademacher [8] and Sloot [12] for horizontal transport. Ng et al. [9] carried out verifications for a single vibration angle of $45^{\circ}$ : for horizontal transport the experiments agree best with the theory for this vibration angle.

\section{Extensions of the point mass theory}

In an attempt to resolve the observed discrepancies between experiments and theory for throw numbers around 1.5 to 2 , the following three modifications were briefly investigated:

(i) By measuring the accelerations of the trough it was found that they were not purely sinusoidal, although the displacement signals were sinusoidal in both directions. In order to investigate the sensitivity to noise in the accelerations of the predicted velocity efficiency, a numerical program was developed, similar to that of $\mathrm{Ng}$ et al. [9]. In the simulation the trough was subjected to an acceleration with a high order 
Table 1

Influence of the restitution coefficient on the velocity efficiency $(\tan \beta$ $=0.25 ; \mu=0.23$ )

\begin{tabular}{clcl}
\hline $\tan \alpha$ & $e$ & $\eta$ for $\Gamma=1.5$ & $\eta$ for $\Gamma=2.0$ \\
\hline-0.05 & 0 & 0.477 & 0.537 \\
& 0.1 & 0.477 & 0.827 \\
& 0.2 & 0.477 & 0.858 \\
0 & 0 & 0.210 & 0.517 \\
& 0.1 & 0.210 & 0.793 \\
& 0.2 & 0.210 & 0.838 \\
+0.05 & 0 & -0.065 & 0.498 \\
& 0.1 & -0.065 & 0.819 \\
& 0.2 & 0.249 & 0.819 \\
\hline
\end{tabular}

(k) artificial noise with (dimensionless) amplitude $a$ and phase shift $\phi$ :

$\ddot{X}=\ddot{Y}=-\sin \omega t-a \sin (k \omega t+\pi)$

Calculating some efficiencies with different values of $a$ and phase shifts $\phi$, the variation remained within $10 \%$ for $a \leq 0.2$ and $k \approx 25$ (which is the order of measured noise). This means that the large difference between theory and experiments can not be explained by the deviation in trough acceleration from the sinusoidal shape.

(ii) The rotation of the particles could lead to an increase of the relative displacement due to their possibly turning over during a collision of a particle with the trough. This would lead to a maximum increase of the relative displacement in the order of a particle diameter. Since trough displacements of around $12 \mathrm{~mm}$ per period were used in the experiments, this influence can be neglected for PVC grains and spinach seed $(d \leq 3.5 \mathrm{~mm})$.

(iii) Van Kappel [13] investigated damping within the layer of granular material during the transport in vibratory conveyors from a theoretical and experimental point of view. In order to include a more sophisticated model for the collision, a partly elastic collision is assumed here. The relation between the normal velocities of the point mass before and after the collision is defined by means of a coefficient of restitution $e$ [14]:

$e \equiv-\frac{\dot{y}_{\mathrm{rel}}\left(\epsilon_{\mathrm{F}}^{+}\right)}{\dot{y}_{\mathrm{rel}}\left(\epsilon_{\mathrm{F}}^{-}\right)}$

Note that a purely plastic collision corresponds to $e=0$. The mass term in Eq. (17a) is now replaced by $m(1+e)$; the consequence of this is that in Eq. (18), $\mu$ is replaced by $\mu(1+e)$. Now the system can be periodical over a period of more than $2 \pi$ or even not periodical at all. The velocity efficiency was determined by calculating an average displacement over a long time interval. The influence of a partly elastic collision on the velocity efficiency is shown in Table 1 .

Note the large increase of the velocity efficiency for a throw number of 2.0 , even for a small coefficient of restitution. Although the large deviations for throw numbers of around 1.5 are not fully explained, this theoretical refinement of the original point mass theory forms a promising approach to further research of the transport of granular materials by vibratory conveyors. For further research it is also recommended to include air drag into the theory and to determine the coefficient of restitution experimentally.

The presented point mass theory does not take into account the interactions between the particles of the granular material. By simulating the behaviour of all particles simultaneously, a so-called discrete element method simulation, these interactions can be properly accounted for. Hogue and Newland [15] give results of such an approach in their study of the sieving process.

\section{Conclusions}

A point mass theory is presented for the rest, slide and flight phases that occur during the transport of granular materials by inclined vibratory conveyors. Plots are given, showing the influence of inclination, throw number, friction coefficient and vibration angle on the velocity efficiency. When the vibration angle approaches 0 , the velocity effciency is calculated analytically for slide conveyors.

A method is described in detail for determining the coefficient of friction between granular material and trough as a function of the measured maximum relative displacement on a horizontal trough.

Experiments were performed with PVC grains and spinach seed for inclined and declined transport. For slide conveyors the agreement in velocity efficiency between theory and the experiments was satisfactory. For flight conveyors the agreement was fairly good for throw numbers of about 2.5 . For throw numbers between 1.5 and 2.0 the experiments showed a velocity efficiency that was almost independent of the vibration angle, contrary to what is predicted by the theory.

In order to find an explanation for these deviations, some possibilities were investigated. This includes the effect of a partly elastic collision. For small coefficients of restitution $(0.1-0.2)$ the theory coincides quite well with the experimental data for throw numbers around 2.0.

\section{List of symbols}

C constant in Eq. (20) (-)

d diameter $(\mathrm{m})$

$e \quad$ coefficient of restitution (-)

$F_{\mathrm{w}} \quad$ friction force $(\mathrm{N})$

$g \quad$ acceleration due to gravity $\left(\mathrm{m} \mathrm{s}^{-2}\right)$

$k \quad$ order of artificial noise (-)

$K \quad$ machine number (-)

$m \quad$ mass $(\mathrm{kg})$

$N \quad$ normal force $(\mathrm{N})$

$n \quad$ number of periods per flight (-)

$r \quad$ radius, amplitude of displacement in direction of vibration (m) 
$s \quad$ displacement in vibrational direction $(\mathrm{m})$

$t \quad$ time (s)

$T \quad$ period (s)

$\bar{v} \quad$ average tangential material velocity $\left(\mathrm{m} \mathrm{s}^{-1}\right)$

$v_{\mathrm{F}} \quad$ tangential material velocity at the start of the flight $\left(\mathrm{m} \mathrm{s}^{-1}\right)$

$\begin{array}{ll}x & \text { coordinate tangential to trough }(\mathrm{m}) \\ X & \text { dimensionless coordinate tangential to trough }\end{array}$

$(-)$

$y \quad$ coordinate normal to trough ( $\mathrm{m}$ )

$Y \quad$ dimensionless coordinate normal to trough (-)

\section{Greek letters}

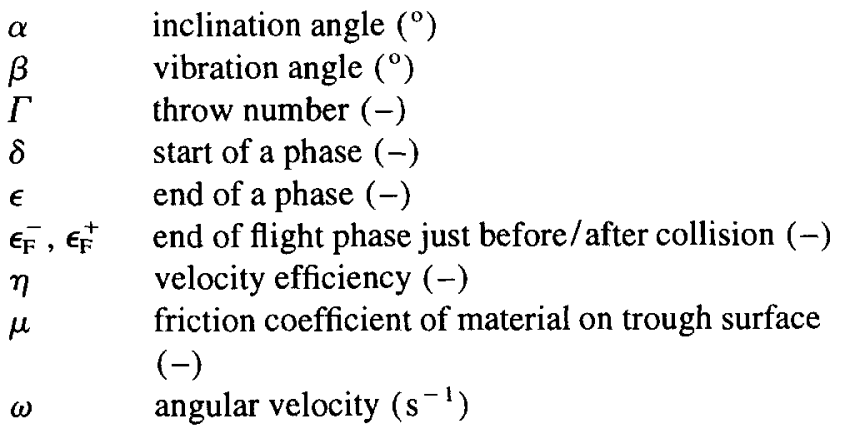

\section{Subscripts}

C collision

F flight

$i \quad$ index according to Eq. (8) $(i=1,3)$

m material or point mass

$\mathrm{N}$ negative slide phase

$\begin{array}{ll}\mathrm{N}^{\prime} & \text { negative slide phase after collision } \\ \mathrm{P} & \text { positive slide phase } \\ \mathrm{P}^{\prime} & \text { positive slide phase after collision } \\ \text { rel } & \text { relative (material-trough) } \\ \mathrm{t} & \text { trough or conveyor }\end{array}$

\section{Acknowledgements}

The authors would like to thank Professor Dr. Ir. F.J.C. Rademacher (Rademacher Engineering, Borne, Netherlands), for the fruitful discussions and pleasant cooperation.

\section{References}

[1] J.H. Booth and H. McCallion, Proc. Inst. Mech. Eng., 178 (1963) 521.

[2] R.M. Nedderman and G.H.L. Harding, Trans. Inst. Chem. Eng., 68 (1990) 123.

[3] G. Pajer, H. Kuhnt and F. Kurth, Fördertechnik-Stetigförderer, VEB Verlag Technik, Berlin, 3rd edn., 1977, pp. 230-235.

[4] K. Erdesz and A. Szalay, Powder Technol., 55 (1988) 87.

[5] K. Erdesz and J. Németh, Powder Technol., 55 (1988) 161.

[6] S.P. Hota and R. Karmaker, Bulk Solids Handling, 8 (1988) 715.

[7] F.J.C. Rademacher, Vibratory Conveyors, Lecture Notes, University of Twente, Enschede, Netherlands, 1986 (in Dutch).

[8] F.J.C. Rademacher and L. ter Borg, Eng. Res., 60 (1994) 261.

[9] K.L. Ng, L.A. Ang and S.C. Chng, Proc. Inst. Mech. Eng., 200 (1986) 123.

[10] G.H. Lim, Adv. Eng. Software, 18 (1993) 53.

[11] S.P.E. Persson and M.K. Megnin, Trans. ASAE, 35 (1992) 395.

[12] E.M. Sloot, M.Sc. Thesis, University of Twente, Enschede, Netherlands, 1992 (in Dutch).

[13] J.M. van Kappel, M.Sc. Thesis, University of Twente, Enschede, Netherlands, 1991 (in Dutch).

[14] R.M. Brach, J. Appl. Mech., S6 (1989) 133.

[15] C. Hogue and D. Newland, Powder Technol., 78 (1994) 51. 